\title{
Performance Analysis and Comparison of Reactive and Proactive MANET Routing Protocols
}

\author{
Lavneet Bansal \\ Assistant Professor \\ Department of Computer Engineering \\ MIMIT, Malout
}

\author{
Swati Jindal \\ M.Tech Student \\ Department of Computer Engineering \\ Guru Nanak Dev University, Amritsar
}

\begin{abstract}
Mobile Ad hoc networks are characterized by multihop wireless connectivity, frequently changing network topology and the need for efficient dynamic routing protocols plays an important role. We compare the performance of three prominent Reactive protocols TORA,DSR and proactive protocol DSDV for different scenarios i.e. pause time, simulation time and no of nodes An extensive simulation is performed using NS-2 simulator and end to end delay, packet delivery ratio, packet loss ratio, throughput and jitter,Normalized Routing load. In this paper At the end it is concluded that in case of TCP DSDV(Proactive) is best and in case of CBR DSR(Reactive) is best. Performance of TORA is average in all cases except packet loss.TORA has maximum Normalized Routing load.
\end{abstract}

Keywords: TORA, DSR, DSDV, MANET, protocols, NS2 simulator

\section{INTRODUCTION}

Ad-hoc networks are the key factor in the evaluation of wireless communication quoted as corner stones of future generation wireless networking. Wireless LANs support user demand for flawless connectivity, flexibility, and mobility. Generally there are two distinct approaches for enabling wireless mobile units to communicate with each other:

Infrastructure-based - Wireless mobile networks have traditionally been based on the cellular concept and relied on good infrastructure support.

Infrastructure-less - Infrastructure-less approach, the mobile wireless network is commonly known as a mobile ad hoc network (MANET) i.e. collection of wireless nodes that can dynamically form a network to exchange information without using any preexisting fixed network infrastructure.

A fundamental problem in ad hoc networking is routing i.e. how to deliver data packets among MNs efficiently without predetermined topology or centralized control, which is the main objective of ad hoc routing protocols. Since mobile ad hoc networks change their topology frequently, routing in such networks is a challenging task. Moreover, bandwidth, energy and physical security are limited. The Mobile Ad-Hoc Network is characterized by energy constrained nodes, bandwidth constrained links and dynamic topology. Potential applications for this class of network includes instant network infrastructure to support collaborative computing in temporary or mobile environments, emergency rescue networks for disaster management, remote control of electrical appliance, communication systems such as IVC (Inter-Vehicle Communications), and mobile access to the global Internet. Routing protocols for Ad-hoc networking can be classified into four categories viz. (i) Based on routing information update routing mechanism (proactive or table-driven, reactive or on-demand and hybrid protocols), (ii) Based on the use of Temporal information (Past Temporal and Future Temporal) for routing, (iii) Based on routing topology (Flat Topology, Hierarchical Topology), (iv) Based on the Utilization of Specific Resources (Power Aware Routing and Geographical Information Assisted Routing) [29].

\subsection{Destination-Sequenced Distance-Vector Routing [DSDV] [6]:}

The Table-driven DSDV is a proactive protocol that is modified version of the Distributed Bellman-Ford (DBF) Algorithm that was used successfully in many dynamic packet switched networks The design goals of DSDV were to keep the simplicity of the distributed Bellmann -Ford and to avoid the looping problem in routing tables using the concept of sequence number. It uses full dump and update increment to lessen the traffic load. The improvement made in this is the avoidance of infinite loop. In DSDV, each node is required to transmit a sequence number that is linked to destination usually originated by owner , at which is periodically increased by two and transmitted along with any other routing update messages to all neighboring nodes .A non-owner node updates a sequence number of a route is when it detects a link break on that route. Owner nodes uses even numbers and non owner nodes uses odd numbers as sequence number.

\subsection{Dynamic Source Routing Protocol (DSR) [7]:}

The Dynamic Source Routing (DSR) protocol is an ondemand reactive unicast routing protocol based on source routing. DSR protocol is composed by two "on-demand" mechanisms, which are requested only when two nodes want to communicate with each other. In DSR, each node uses buffer technology to keep route information of all the nodes. There are two major phases in DSR such as:

\section{- Route discovery \\ - Route maintenance}

In DSR, every mobile node in the network needs to maintain a route cache where it caches source routes that it has learned. When a host wants to send a packet to some other host, it first checks its route cache for a source route to the destination. In the case a route is found, the sender uses this route to propagate the packet. Otherwise the source node initiates the route discovery process. Route discovery and route maintenance are the two major parts of the DSR protocol. 


\subsection{Temporally Ordered Routing Algorithm (TORA):}

TORA (Temporally ordered Routing Algorithm) is an ondemand distributed routing protocol which uses a reversal algorithm and designed for route initiated by source nodes or rather, on demand and provide loop free and multiple routes(to lessen congestion) and it establish route quickly and minimize the overhead while communication .Moreover, it is desirable to detect network partition and delete invalid routes. TORA is unique by maintaining multiple routes to a destination .It also still maintain state on a per destination basis. However the shortest route paths are considered less important so preference is given to longer routes to minimize the overhead. It does not work well in low mobility networks.

\section{RELATED WORK}

Analysis and simulation of wireless AD-Hoc network routing was done by Mohamed et al [2004][1]. The simulation results show that AODV and DSDV can be used for most of ad-hoc applications delivering about $95 \%$ of data packets to the destination nodes.Performance analysis of three routing protocols(DSDV,AODV and DSR) in wireless mobile Ad Hoc networks were discussed by Lakshmi et al [2006][2]. It was analyzed that DSDV are more suitable for small networks where changes in the topology are limited. Chang et al [2006] $[53,3]$ evaluated that AODV and DSR, both show better performance than the other in terms of certain metrics.Jayakumar et al [2007][4] concluded that for DSR and AODV, packet delivery ratio is independent of offered traffic load, with both protocols delivering between $85 \%$ and $100 \%$ of the packets in all cases.Kumar et al [2008]5] analyzed that Both reactive protocols performed well in high mobility scenarios than proactive protocol.Malany et al[2009][47,13] has done the Throughput and Delay Comparison and faced the problem of switching off of the scenario for higher node densities. It might be due to the processor capability (RAM usage).Karthik et al [2010][44,9] investigated that The performance of the DSR and AODV is superior to the DSDV in conformance.Manickam1 et al [2011][10] analyzed that DSDV produces low end-to-end delay compared to other protocols. Ambhaikar et al[2011][11] has analyzed that the performance of AODV protocol is better than the DSDV protocol. AODV performance is the best considering its ability to maintain connection by periodic exchange of information.Karthiga et al[2011][12] has observed that DSDV performance is best considering its ability to maintain connection by periodic exchange of information, which is required for TCP, based traffic.

\section{SIMULATION AND PERFORMANCE ANALYSIS}

In this dissertation, we have taken three different scenarios. In scenarios five different nodes i.e. 100, 80, 60, 40, 30, different pause time i.e. 5,10,15,20 (sec) and simulation time i.e. 100, 75,50 (sec) have been taken based on TCP based traffic pattern and CBR based traffic pattern.

Table 3.1: Simulation Parameters for test scenario 1

\begin{tabular}{|l|l|}
\hline Parameter & Value \\
\hline Number of nodes & $100,80,60,40,30$ \\
\hline Maximum Speed & $20 \mathrm{~m} / \mathrm{s}$ \\
\hline Simulation time & $100 \mathrm{sec}$ \\
\hline Pause Time & $20 \mathrm{sec}$ \\
\hline Environment Size & $1000 x 1000$ \\
\hline Packet Size & 512 bytes \\
\hline Traffic Type & TCP \\
\hline Packet Rate & 8 packet/sec $(1 \mathrm{kbps})$ \\
\hline
\end{tabular}

Table 3.2: Simulation Parameters for test scenario 2

\begin{tabular}{|l|l|}
\hline Parameter & Value \\
\hline Number of nodes & 80 \\
\hline Maximum Speed & $20 \mathrm{~m} / \mathrm{s}$ \\
\hline Simulation time & $100 \mathrm{sec}$ \\
\hline Pause Time & $5,10,15,20 \mathrm{sec}$ \\
\hline Environment Size & $1000 \times 1000$ \\
\hline Packet Size & 512 bytes \\
\hline Traffic Type & TCP \\
\hline Packet Rate & 8 packet $/ \mathrm{sec}(1 \mathrm{kbps})$ \\
\hline
\end{tabular}

Table 3.3: Simulation Parameters for test scenario 3

\begin{tabular}{|l|l|}
\hline Parameter & Value \\
\hline Number of nodes & 80 \\
\hline Maximum Speed & $20 \mathrm{~m} / \mathrm{s}$ \\
\hline Simulation time & $100,75,50 \mathrm{sec}$ \\
\hline Pause Time & $15 \mathrm{sec}$ \\
\hline Environment Size & $1000 \times 1000$ \\
\hline Packet Size & 512 bytes \\
\hline Traffic Type & TCP \\
\hline Packet Rate & 8 packet/sec $(1 \mathrm{kbps})$ \\
\hline
\end{tabular}

Table 3.4: Simulation Parameters for test scenario 4

\begin{tabular}{|l|l|}
\hline Parameter & Value \\
\hline Number of nodes & $100,80,60,40,30$ \\
\hline Maximum Speed & $20 \mathrm{~m} / \mathrm{s}$ \\
\hline Simulation time & $100 \mathrm{sec}$ \\
\hline Pause Time & $5.0 \mathrm{sec}$ \\
\hline Environment Size & $1000 x 1000$ \\
\hline Packet Size & 512 bytes \\
\hline Traffic Type & CBR \\
\hline Packet Rate & 8 packet/sec $(1 \mathrm{kbps})$ \\
\hline
\end{tabular}

Table 3.5: Simulation Parameters for test scenario 5

\begin{tabular}{|l|l|}
\hline Parameter & Value \\
\hline Number of nodes & 80 \\
\hline Maximum Speed & $20 \mathrm{~m} / \mathrm{s}$ \\
\hline Simulation time & $100,75,50 \mathrm{sec}$ \\
\hline Pause Time & $20.0 \mathrm{sec}$ \\
\hline Environment Size & $1000 \times 1000$ \\
\hline Packet Size & 512 bytes \\
\hline Traffic Type & Constant Bit Rate \\
\hline Packet Rate & 8 packet/sec $(1 \mathrm{kbps})$ \\
\hline
\end{tabular}

Table 3.6: Simulation Parameters for test scenario 6

\begin{tabular}{|l|l|}
\hline Parameter & Value \\
\hline Number of nodes & 80 \\
\hline Maximum Speed & $20 \mathrm{~m} / \mathrm{s}$ \\
\hline Simulation time & $100 \mathrm{sec}$ \\
\hline Pause Time & $5,10,15,20 \mathrm{sec}$ \\
\hline Environment Size & $1000 x 1000$ \\
\hline Packet Size & 512 bytes \\
\hline Traffic Type & Constant Bit Rate \\
\hline Packet Rate & 8 packet/sec $(1 \mathrm{kbps})$ \\
\hline
\end{tabular}

\section{RESULTS}

Performance comparisons have been made between TORA, DSR and DSDV protocols. Identical mobility and traffic scenarios are used across protocols to gather fair results.In this it is analysed that end to end delay is less for proactive 
protocol ie DSDV,packet delivery ratio is high in case of DSR.Throughput is high for DSR in CBR and DSDV in TCP. Tora has minimum packet Loss.jitter is more in case of TCP than CBR.

\subsection{Impact On End To End Delay}

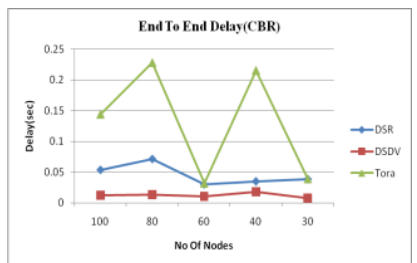

Figure 4.1:End to End Delay by varying no of nodes in CBR

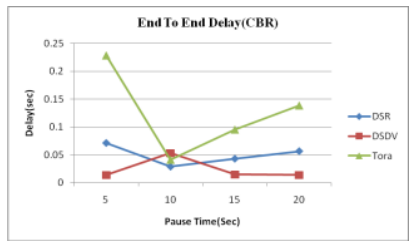

Figure 4.2 End to End Delay by varying pause time in CBR

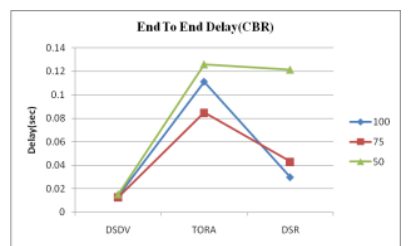

Figure 4.3 End to End Delay by varying simulation Time in CBR

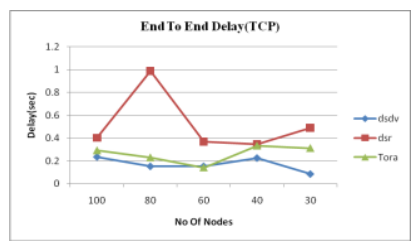

Figure 4.4 End to End Delay by varying no of nodes in TCP

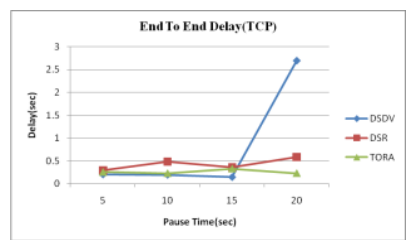

Figure 4.5 End to End Delay by varying pause time in TCP

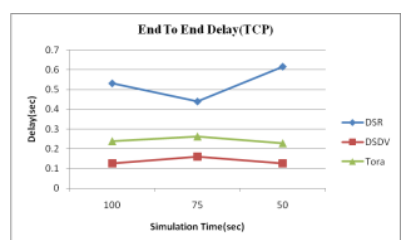

Figure 4.6 End to End Delay by varying simulation time in TCP

- In CBR TORA has maximum delay and DSDV has minimum.

- In case of TCP DSDV has minimum delay and DSR has maximum Delay in all cases and TORA has average delay.
- So DSDV has minimum delay because it's a proactive protocol

\subsection{Impact On Packet Delivery Ratio}

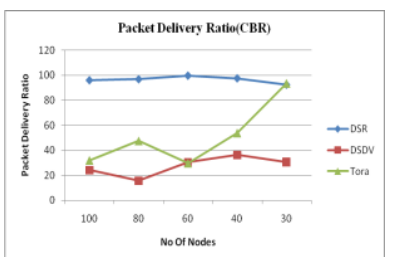

Figure 4.7 Packet Delivery Ratio by varying no of nodes in CBR

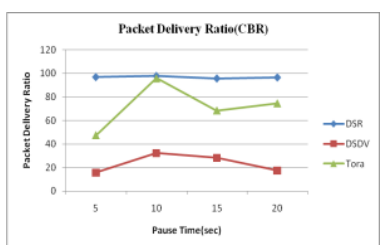

Figure 4.8 Packet Delivery Ratio by varying pause time in CBR

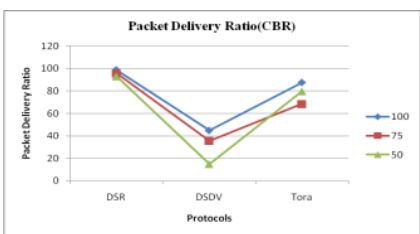

Figure 4.9 Packet Delivery Ratio by varying simulation time in $\mathrm{CBR}$

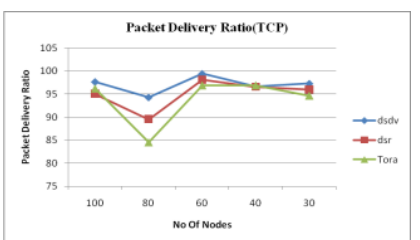

Figure 4.10 Packet Delivery Ratio by varying no of nodes in TCP

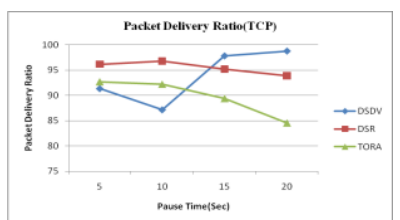

Figure 4.11 Packet Delivery Ratio by varying Pause Time in TCP

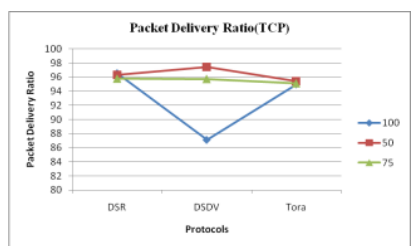

Figure 4.12 Packet Delivery Ratio by varying simulation time in TCP

- We conclude that in case of packet delivery Ratio DSR is best for both traffic pattern. 


\subsection{Impact On Throughput}

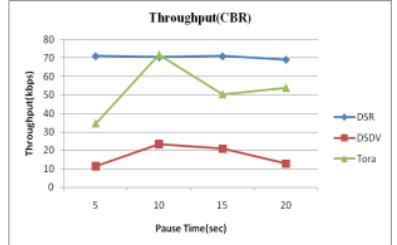

Figure 4.13 Throughput by varying Pause time in CBR

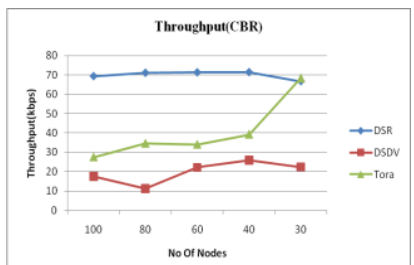

Figure 4.14 Throughput by varying no of nodes in CBR

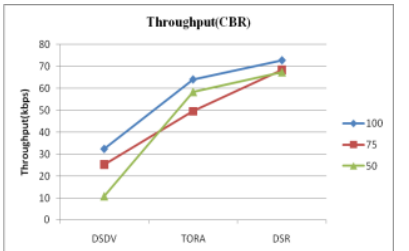

Figure 4.15 Throughput by varying simulation time in CBR

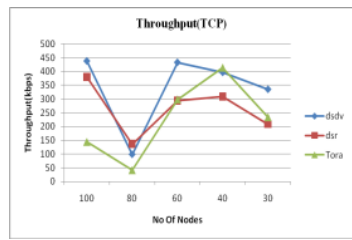

Figure 4.16 Throughput by varying No of nodes in TCP

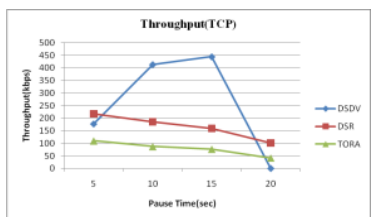

Figure 4.17 Throughput by varying Pause time in TCP

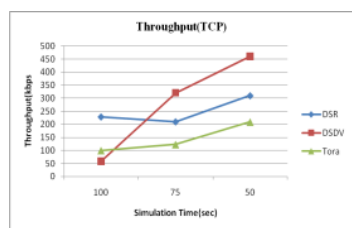

Figure 4.18 Throughput by simulation time in TCP

- In case of CBR DSR has maximum throughput and DSDV has minimum.

- In case of TCP DSDV has the maximum value of throughput in all cases. but overall In case of TCP DSR has average throughput and TORA has minimum.

- As the no of nodes increases throughput also increases in case of CBR.

\subsection{Impact On Packet Loss}

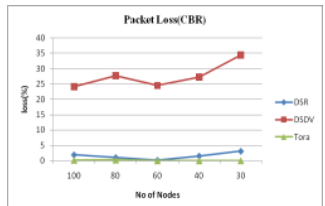

Figure 4.19 Packet loss by varying no of nodes in CBR

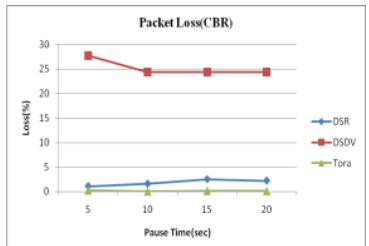

Figure 4.20 Packet loss by varying pause time in CBR

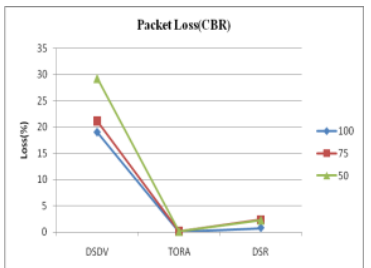

Figure 4.21 Packet Loss by varying simulation time in CBR

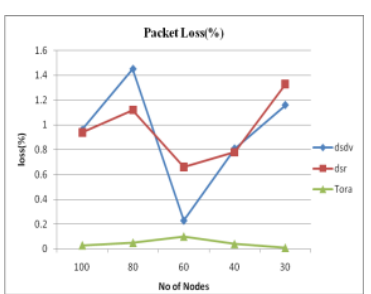

Figure 4.22 Packet Loss by varying no of nodes in TCP

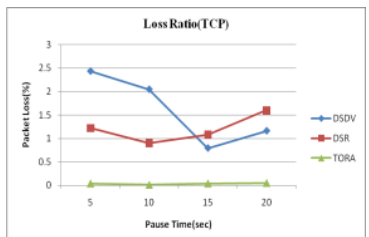

Figure 4.23 Packet Loss by varying Pause time in TCP

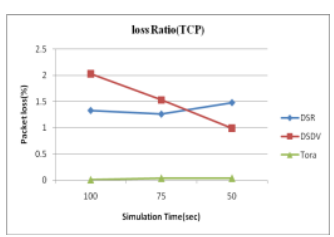

Figure 4.24 Packet Loss by varying simulation time in TCP

- In case of CBR and TCP Traffic pattern DSDV has maximum loss and TORA has minimum loss Ratio. 


\subsection{Impact On Jitter}

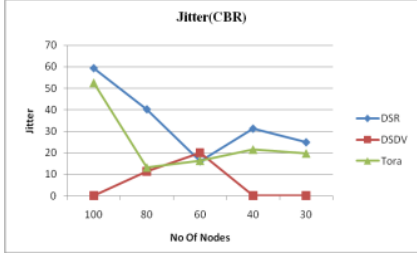

Figure 4.25 Jitter by varying no of nodes in CBR

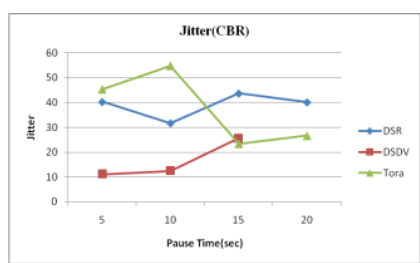

Figure 4.26 Jitter by varying pause time in CBR

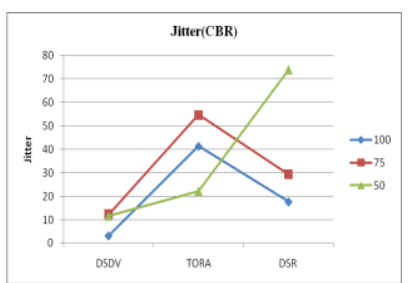

Figure 4.27 Jitter by varying simulation time in CBR

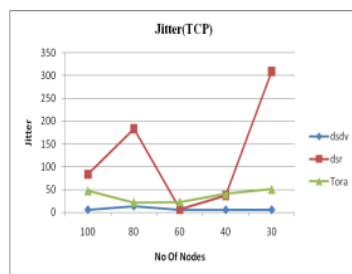

Figure 4.28 Jitter by varying no of nodes in TCP

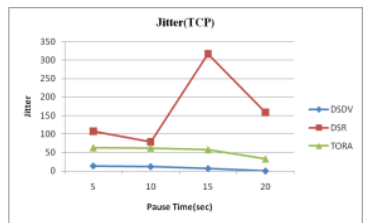

Figure 4.29 Jitter by varying pause time in TCP

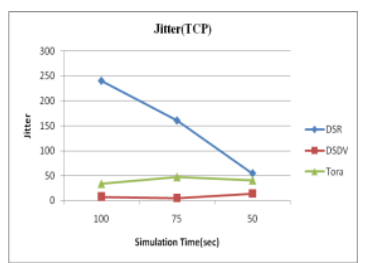

Figure 4.30 Jitter by varying simulation time in TCP

- Jitter is more in case of TCP than in CBR traffic pattern.

\subsection{Normalized Routing Overload}

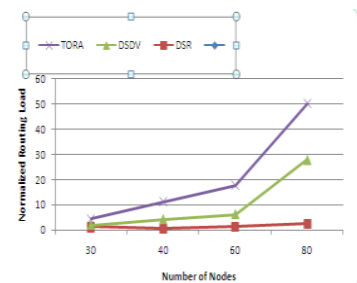

Figure 4.31 Normalized Routing Load by varying no of nodes in $\mathrm{CBR}$

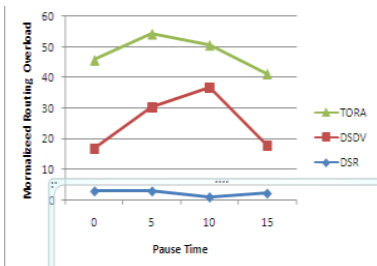

Figure 4.32 Normalized Routing Load by varying Pause Time in $\mathrm{CBR}$

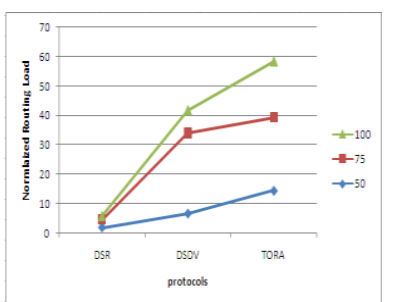

Figure 4.33 Normalized Routing Load by varing simulation time in CBR

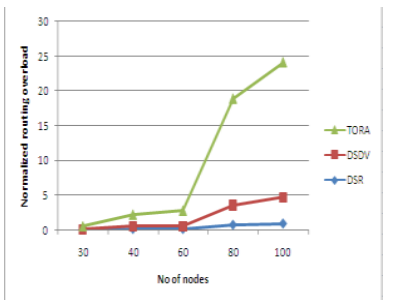

Figure 4.34 Normalized Routing Load by varying no of nodes in TCP

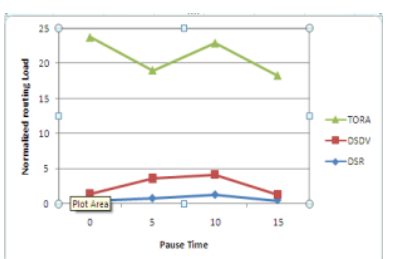

Figure 4.35 Normalized Routing Load by varying Pause time in TCP

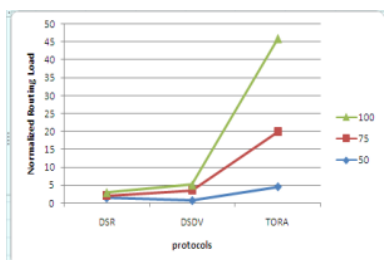

Figure 4.36 Normalized Routing Load by varying simulation time in TCP 
- In case of TCP,CBR TORA has maximum routing Overload.

\section{CONCLUSIONS \& FUTURE SCOPE}

In case of TCP DSDV is best in end to end delay, throughput, jitter. In case of TCP and CBR DSR is best in packet delivery ratio. This is due to the fact that in DSDV the routing table exchanges would increase with larger number of nodes. And DSR is best in case of throughput in CBR traffic pattern. Packet loss Ratio is less in TORA for both CBR and TCP traffic patterns. At the end it is concluded that in case of TCP DSDV (Proactive) is best and in case of CBR DSR (Reactive) is best. Performance of TORA is average in all cases except packet loss. In particular, DSR uses source routing and route caches, and does not depend on any periodic or timer-based activities. DSR exploits caching aggressively and maintains multiple routes per destination.

During present work, impact of VBR traffic was also tried and studied for all three protocols using NS2 but it didn't worked out as NS2 does not support VBR traffic. It was observed that VBR traffic can be studied using another simulator GloMoSim which supports this type of traffic and the results of VBR traffic can be validated by comparing with results of CBR traffic.

\begin{tabular}{|l|l|l|l|}
\hline PROTOCOLS & TORA & DSR & DSDV \\
\hline DELAY(TCP) & 2 & 1 & 3 \\
\hline PD RATIO(TCP) & 2 & 3 & 1 \\
\hline THROUGHPUT(TCP) & 1 & 2 & 3 \\
\hline JITTER(TCP) & 2 & 1 & 3 \\
\hline PACKET LOSS(TCP) & 3 & 2 & 1 \\
\hline ROUTING OVERLOAD & 1 & 2 & 3 \\
\hline TOTAL & 11 & 11 & 14 \\
\hline
\end{tabular}

Table5.1: Numerical Comparison Of The Three Routing Protocols For TCP.

\begin{tabular}{|l|l|l|l|}
\hline PROTOCOLS & TORA & DSR & DSDV \\
\hline DELAY(CBR) & 1 & 2 & 3 \\
\hline PD RATIO(CBR) & 1 & 3 & 2 \\
\hline THROUGHPUT(CBR) & 2 & 3 & 1 \\
\hline JITTER(CBR) & 2 & 1 & 3 \\
\hline PACKET LOSS(CBR) & 3 & 2 & 1 \\
\hline ROUTING OVERLOAD & 1 & 3 & 2 \\
\hline TOTAL & 9 & 11 & 10 \\
\hline
\end{tabular}

Table5.2: Numerical Comparison Of The Three Routing Protocols FOR CBR.

\section{REFERENCES}

[1] Abdul Mohamed Bashir Haimid" Analysis and Simulation of wireless Ad-hoc network routing protocols", April 2004.

[2] M Lakshmi and P.E Sankaranarayanan," Performance Analysis of three Routing Protocols in Wireless Mobile Ad Hoc networks ", Information Technology Journal 5(1): 114-120,2006, ISSN 1812-5638,

[3] Chang Zhijiang, Gaydadjiev Georgi, Vassiliadis Stamatis," Routing Protocols for Mobile Ad-hoc Networks: Current Development and Evaluation" Computer Engineering laboratory, EEMCS, Delft University of Technology.

[4] G Jayakumar. and G Gopinath, "Ad Hoc Mobile Wireless Networks Routing Protocols- A Review," Journal of Computer Science, Vol. 3, No.8, pp. 574-582, 2007.

[5] A Kumar, B.R Lokanatha C Reddy. and S Prakash.Hiremath," IJCSNS International Journal of Computer Science and Network Security, VOL.8 No.6, June 2008..

[6] C. E. Perkins and P.Bhagwat "Highly Dynamic Destination-Sequenced Distance-Vector Routing (DSDV) for Mobile Computers", SIGCOMM 94 -8/94 London England UK @ 1994 ACM 0-89791 -6824/94/0008.

[7] J.Broch, D.Johnson and D., Maltz "The Dynamic Source Routing Protocol for mobile ad hoc October 1999.

[8] R Huang, Y Zhuang, Q Cao., "Simulation and Analysis of Protocols in Ad Hoc Network", 2009 International Conference on Electronic Computer Technology (C) 2009 IEEE

[9] S.Karthik ,S.Kannan ,Arunachalam ,A.P Ravichandran T,,Valarmathi,"An Investigation about Performance Comparison of Multi-Hop Wireless Ad-Hoc Network Routing Protocols in MANET " in IJCSI International Journal of Computer Science Issues, Vol. 7, Issue 3, No 6, May 201035 ISSN (Online): 1694-0784.

[10] P Manickam,T.Guru Baskar, M Girija , Manimegalai $\mathrm{D}$," Performance comparison of routing protocols in mobilr Ad hoc networks ", International Journal of Wireless \& Mobile Networks (IJWMN) Vol. 3, No. 1, February 2011 DOI : 10.5121/ijwmn.2011.3109 98.

[11] A Ambhaikar, D Mitra,R.Deshmukh, " Performance of MANET Routing Protocol for Improving Scalability ", Published in International Journal of Advanced Engineering \& Application, Jan 2011 Issue.

[12] G.Karthiga,J.Benitha,Chandir Moses Jeban," Performance Analysis of Various Ad-Hoc Routing Protocols in Multicast Environment", Department of CSE, Karunya University, Coimbatore, Tamilnadu, India.

[13] B Boomarani Malany, V.R Sarma Dhulipala, and R.M.Chandrasekaran, "Throughput and Delay Routing Protocols" ,Comparison of MANET Int. J. Open Problems Compt. Math., Vol. 2, No. 3, September 2009 ISSN 1998-6262; Copyright @ICSRS Publication, 2009 www.i-csrs.org. 